\title{
El marco jurídico y la política de comunicación social en el marco del TLC
}

\author{
María de la Luz Casas Pérez \\ ITESM Campus Morelos
}

\section{Introducción}

El presente trabajo es parte de un proyecto de investigación llevado a cabo entre 1994 y 1996 con colegas interesados en el desarrollo de las industrias audiovisuales en México y Canadá, y especialmente en la provincia francesa de Quebec. Dicho proyecto conocido como Proyecto Monarca tuvo como objetivo realizar una comparación entre ambos sistemas de medios observándolos desde distintos ámbifos. Uno de ellos fue precisamente el de la reglamentación.

Tanto a mexicanos como a canadienses nos interesaba conocer en qué medida nuestros respectivos sistemas políticos estaban haciendo frente a las presiones de un mercado económico integrado y forzados por los compromisos adquiridos a través del Tratado de Libre Comercio de América del Norte (TLC).

Un primer intercambio nos acercó a conocer la estructura jurídica fundamental que rige a ambos sistemas de medios: en primer término descubrimos que prevalecía una estructura mixta (medios privados y medios públicos), regida por una reglamentación que defiende en principio la autonomía de ambos países sobre sus sistemas de comunicacłones y el derecho a la libre expresión. Sin embargo, los mecanismos a través de los cuales ambos países ponen en operación controles para limitar o expander el espectro de los sistemas de comunicación son variados. ${ }^{1}$

1 Gaĕtan Tremblay et Jean-Guy Lacroix. Le Projet Monarque. Étude comparée des industries québécoises et mexicaines de láudiovisuel. Collection DocumentsGricis. Université du Québec à Montréal, 1995. Ver especificamente los trabajos sobre reglamentación en medios audiovisuales, pp. 13-50. 
Un segundo trabajo se dedicó a tratar de entender precisamente los modos como la industria de medios, hace uso de sus privilegios en la estructuración de los sistemas de comunicación a nivel nacional. En el caso de Mèxico, se analizó la forma en la que se recomponen las fuerzas económicas y políticas que rigen los sistemas de comunicaciones en México, particularmente a la luz de un cambio de gobierno y de una crisis económica que, aunque iniciada en México, repercute a todo lo largo y ancho del continente.

Lo anterior desde luego incide directamente en la forma en que operan los sistemas de comunicación mexicanos, ajustándolos tanto a las condiciones estructurales del país como a las nuevas directrices de los mercados internacionales. Así por ejemplo se observa cómo los mercados que mayormente sufren una recomposición, son el de las telecomunicaciones, (incluídas la telefonía, los servicios de información, internet y otros) y el de la televisión por cable.

En el presente trabajo se analizan los esquemas de comunicación a los que faculta el cuerpo del Tratado de Libre Comercio de América del Norte (TLC) para los países firmantes, haciendo especial énfasis en el caso de México. En segundo lugar, se lleva a cabo una revisión de intentos de modificación a la reglamentación en medios de comunicación que se dieron en México entre 1995 y 1996. En tercer lugar, una comparación entre los mecanismos de consulta pública en materia de comunicaciones llevados a cabo recientemente, con el propósito de debatir los retos a los que se enfrenta el diseño de políticas públicas en el sector e incluso proponer los compromisos sociales a los que se deberá apegar la futura actividad de comunicaciones.

\section{El TLC y los medios}

En el caso de México, y seguramente también en el caso de Canadá, las implicaciones del Tratado de Libre Comercio de América del Norte (TLC) en torno a los medios de comunicación y al sector de servicios de información de valor agregado, se dejarán sentir por mucho tiempo.

Las fuerzas que condujeron tanto a México como a Canadá a integrar el bloque económico de América del N̦orte, los intereses empresariales que han empujado a la liberalización de los mercados y el destino que correrán los sistemas de comunicación existentes, son cuestiones que deben ser cuidadosamente reflexionadas. Aquí simplemente nos permitimos hacer una relación de los elementos que aparecen acotados en el cuerpo del documento oficial y que deben ser tomados en consideración para futuros análisis. 
Nos resultan de particular interés no sólo los acuerdos a los que se llega y la forma en la que éstos cristalizan dentro cuerpo del Tratado, sino también sus implicaciones económicas, políticas, sociales y culturales. Es evidente que en gran medida el crecimiento de la economía mexicana era percibido como dependiente del crecimiento en el sector de las comunicaciones. Ya en 1992 el propio Secretario de Comercio de México, encargado de la parte negociadora mexicana, advertía de la importancia del sector servios de información, tales como el de las telecomunicaciones para la economía de la región. ${ }^{2}$ El texto del Tratado de Libre Comercio de América del Norte (TLC), define como actividad de telecomunicaciones a toda aquella transmisión y recepción de señales por cualquier medio electromagnético, y como red o servicio cualquier red pública de telecomunicaciones. Asimismo, el Tratado de Libre Comercio de América del Norte establece como servicios mejorados o de valor agregado a todo aquel servicio de telecomunicaciones que emplee sistemas de procesamiento computarizado que actúe sobre el formato, contenido, código, protocolo 0 aspectos similares de la información, o que proporcione al cliente información adicional, diferente o reestructurada. ${ }^{3}$ Sin embargo y aunque el Tratado de Libre Comercio de América del Norte no menciona explícitamente a la radio y a la televisión, por lo menos en ese apartado, era evidente que por su íntima vinculación con el sector de las telecomunicaciones, éste incidiría fundamentalmente en su desarrollo.

Sin embargo, el Subsecretario de Comunicaciones y Desarrollo Tecnológico Carlos Mier y Terán declaraba: "No se prevén cambios a la Ley Federal de Radio y Televisión. Los avances tecnológicos en telefonía celular, radiolocalización, radiocomunicación de flotillas via microondas y satélite, así como la televisión de alta definición, no tienen nada que ver con los contenidos y sí en cuanto a los medios de distribución" ${ }^{4}$

En perspectiva, dicha declaración evidenciaba ya claramente o bien una falta de visión, o bien el deseo de hacer un no reconocimiento oficial en cuanto al camino que habría de recorrer el desarrollo de los sistemas tecnológicos y de comunicaciones en los años siguientes, ya que nadie duda en estos momentos que el futuro de la radio y la

2 Entonces se declaraba por ejemplo, que el sector servicios de información representaba más de las dos terceras partes de la economía de la región. Jaime Serra Puche en: Resultados de la negociación del Tratado de Libre Comercio. Comparecencia ante la H. Cámara de Senadores. Septiembre de 1992.

3 Tratado de Libre Comercio de América del Norte. Capítulo xIII. Artículo 1310., SECOFI, México, 1992.

4 "Concluyen negociaciones México-EU sobre telecomunicaciones". en: El Financiero, 13 de agosto de 1992, p. 27. 
El marco jurídico y la política de comunicación ...

televisión esté en las telecomunicaciones. A últimas fechas, sin embargo, el Secretario de Comunicaciones y Transportes Carlos Ruiz Sacristán ha declarado públicamente que es en el renglón de las telecomSunicaciones en el cual se prevé que el país tenga un mayor crecimiento en los próximos años, que se está incentivando la inversión extranjera en el área, dando certezas financieras a los inversionistas y haciendo transparente la reglamentación existente, pero sobre todo en favor de una derregulación del sector. ${ }^{5}$

Otro aspecto que cubre el Tratado de Libre Comercio de América del Norte con evidente relación al sector de los medios de comunicación, es el de la propiedad intelectual y los derechos de autor. El gobierno de México envió en 1993 al Senado diversas propuestas de modificación a la Ley Federal de Derechos de Autor de 1956, misma que había recibido ya algunos cambios en 1963, 1981, $1984^{6}$ con el propósito de adecuar la legislación a lo establecido en el marco del TLC en el renglón de propiedad intelectual. Algunos sectores como el de la industria cinematográfica por ejemplo, siguen argumentando hoy en día que dicha adecuación lejos de proteger los intereses de los productores nacionales los perjudicó ya que otorga mayores facilidades y prerrogativas para la explotación de la obra en favor de distribuidores.?

En relación a la propiedad intelectual por ejemplo, el Tratado de Libre Comercio de América del Norte establece como ilícito civil la recepción, en relación con actividades comerciales, o la ulterior distribución de una señal de satélite codificada portadora de programas, que ha sido descodificada sin la autorización del disgtribuidor legítimo de la señal; o la participación en cualquier actividad prohibida de fabricación, importación,

5 Declaraciones del Secretario de Comunicaciones y Transportes Carlos Ruiz Sacristán al noticiero radiofónico Para Empezar en Stereorey, cadena nacional 19 de septiembre de 1996.

6 Los últimos cambios se refieren precisamente al renglón de las industrias culturales, particularmente videogramas y fonogramas, ampliando los plazos de los derechos patrimoniales de 50 a 75 años después de la muerte del autor. Las modificaciones desconocen hasta cierto punto la tradición mexicana del derecho autoral en favor de los derechos de los productores de los discos o videos, así la legislación mexicana se ponía en concordancia con el TLC al adoptar concepto anglosajón del copyright para fines de comercialización, lo cual es consistente con el ámbito de competencia de la Secretaría de Comercio y Fomento Industrial mientras que el derecho intelectual o derecho autoral como era concebido antes se considera competencia de la Secretaría de Educación Pública. "Ley Federal de Derechos de Autor". Diario Oficial de la Federación. 22 de diciembre de 1993.

7 Actualmente existe un proyecto de una nueva ley sobre la materia, sin embargo hay quienes afirman que el nuevo proyecto lejos de darle certeza juridica a los autores y a las industrias culturales, se convertirá en un instrumento que desaliente la creación, la inversión, el uso y la explotación de las obras, lo cual redundará seguramente en el demérito de la cultura nacional. Ver por ejemplo: Mauricio Jalife Daher y José Luis Caballero Leal. "Proyecto de una nueva Ley de Derechos de Autor: un rumor engargolado", en: El Financiero. 15 de octubre de 1996, p.23. 
venta, arrendamiento o cualquier acto que permita descifrar una señal de satélite codificada portadora de programas, sin autorización del distribuidor legítimo. ${ }^{8}$

Es interesante hacer notar sin embargo, que el cuerpo del texto del Tratado habla de paternidad tecnológica para el distribuidor autorizado de la señal y sólo establece en cuanto a los derechos de autor o a los contenidos, que el Tratado se apega a las disposiciones del artículo 2 del Convenio de Berna. ${ }^{9}$

Los medios de comunicación en específico no son mencionados en el Tratado de Libre Comercio, a excepción de una referencia a las industrias culturales ${ }^{10}$ en torno a las cuales el artículo 302 establece que cualquier medida que se adopte o mantega en relación a ellas sobre trato nacional y acceso de bienes al mercado-desgravación arancelaria se regirá conforme a lo dispuesto por el presente Tratado.

En el transcurso de la negociación y gracias a la intervención decidida de Canadá, es que se logra especificar el concepto de industria cultural para referirse a aquellas personas que lleven a cabo la publicación, distribución, venta o exhibición de películas o video o audio incluída la música impresa. Asimismo establece como parte de las industrias culturales a todas las actividades relacionadas con la radio, televisión, transmisión por cable y los servicios de programación de satélites y redes de transmisión; no obstante dicha aclaración aparece únicamente en uno de los anexos técnicos al cuerpo del Tratado. ${ }^{11}$

Tradicionalmente Canadá había establecido una cierta posición de defensa de sus industrias culturales con relación a los Estados Unidos y buscó que éstas no resultasen tan afectadas por los acuerdos del TLC. Al principio de la negociación, Canadá había

8 Tratado de Libre Comercio para América del Norte SEXTA PARTE. Capítulo XVII. Artículos 1705, 1706 y 1707. Opus. Cit.

9 En ese sentido ha habido un cambio importante por lo que se refería a la legislación anterior que otorgaba la propiedad de la obra al autor.

10 Canadá logró establecer una cláusula de excepción para sus industrias culturales en el cuerpo del TLC como resultado de debates anteriores con los Estados Unidos a resultas de un acuerdo bilateral previo conocido como FTA (Free Trade Agreement) en 1989. El artículo 2004 de dicho acuerdo se refiere a la propiedad intelectual y el articulo 2005 establece algunas excepciones relativas directamente a las industrias culturales. Ver por ejemplo: Marcel Masse. "Excerpts from presentation to the Standing Committee on Culture and Communications"., en: Cinema Canada. 164., May 10, 1990, citado por Edward Connor. "The Department of Communications Under Free Trade Regime". en: Canadian Journal of Communication. Volume 16., Number 2, 1991., pp. 239-261. El artículo 2012 de dicho acuerdo define a las industrias culturales y es prácticamente idéntico a la definición establecida dentro del cuerpo del TLCo NAFTA (North American Free Trade Agreement) de 1993.

11 Tratado de Libre Comercio de América del Norte Tomo I. Anexos 2106 y 2107. Opus. Cit., 1992. 
El marco jurídico y la política de comunicación ...

solicitado que se eliminara el renglón de las industrias culturales del acuerdo trilateral, sin embargo los norteamericanos insistieron en que se incluyeran, ${ }^{12}$ y es que Canadá había logrado una serie de excepciones en la primera ronda bilateral con Estados Unidos en el FTA de 1989 que no estaba dispuesto a perder. Quien a la vista perdió claramente fue México, ${ }^{13}$ puesto que los anexos que especifican dentro del cuerpo del Tratado acciones y excepciones por país, acusan una situación mucho más desfavorable para México que para Canadá. (Ver Anexo de lista de México).

Al final, los canadienses mantuvieron su posición de respeto a las industrias culturales conseguida de antemano por la vía del FTA; de hecho, los términos de la negociación con respecto a las industrias culturales permanecen, así como su definición -aunque algunos sectores intelectuales de la sociedad canadiense hubieran preferido que se reformularan-, mientras que México ni siquiera obtuvo un reconocimiento particular a su cultura mas que dentro de las disposiciones generales del acuerdo que establecen la delimitación clara de no competencia desleal, especialmente en el área de la propiedad intelectual y dentro del sector de las comunicaciones.

Los norteamericanos se encontraban especialmente interesados en invertir en el sector de las comunicaciones en México. Así, el Anexo I de la Lista de México del TLC establece específicamente la posibilidad de inversión directa o indirecta hasta en un 49 por ciento de la participación, particularmente en sistemas de televisión y televisión por cable, y en el Anexo II de la misma lista también se faculta la inversión en servicios de radiodifusión, sistemas de distribución multipunto y televisión de alta definición entre otros. También establece que la reserva de México para la decisión de adoptar o mantener

12 Durante las primeras etapas de la negociación, (es decir durante la redacción del texto básico del Tratado), en una entrevista con un periódico norteamericano, la Ministro de Comercio de los Estados Unidos Carla Hills, afirmó que la cultura tendría que estar en la mesa de las negociaciones, y que Canadá continuaría teniendo ferias y comunicaciones y todo ese tipo de cosas, mientras que el Ministro de Comercio de Canadá Michael Wilson, declaraba que la cultura no estaba sujeta a negociación. Al respecto ver por ejemplo: Colleen Fuller. "Fade to Black: Culture Under Free Trade". en: Canadian Forum, August, 1991., p. 5

13 México fue víctima de establecer una posición poco clara al respecto: En junio de 1991, el Dr. Herminio Blanco, Jefe de la negociación mexicana había declarado que "en principio sí se incluirán las industrias del entretenimiento o culturales como son la radio, la televisión, la elaboración de libros, revistas y arte "pues son sectores productores de servicios y los servicios sí se negociarán", mientras que en agosto, el Secretario de Comercio Jaime Serra Puche afirmaba que tales aspectos no se incluirían por "no ser áreas importantes para el desarrollo nacional y además [porque] la cultura mexicana posee una gran solidez que le viene de la herencia cultural de los mexicanos, por lo que no requiere ser protegida". (Notimex, 25 de junio de 1991 y Excélsior 18 de agosto de 1991, respectivamente. También citados por Javier Esteinou Madrid. "El peso del Tratado de Libre Comercio sobre la cultura nacional". Revista Mexicana de Comunicación. Año cinco, Número veintisiete, enero-febrero de 1993., p. 47). 
cualquier medida en el sector no se aplica a la producción, venta o derechos de programas de radio y televisión, lo cual deja a ese sector totalmente desprotegido y libre para la continuación de importación de material extranjero. (Ver Anexo Lista de México).

Probablemente no sea posible aseverar que las transformaciones sufridas recientemente en el sector de los medios se dieron a resulta de la firma del TLC, aún cuando es evidente que coinciden con una serie de cambios tanto económicos como políticos y sociales. Los cambios sufridos en el sector de las telecomunicaciones y de los medios de comunicación social han sido más que notorios: un aumento en la oferta televisiva y radial, el incremento de los servicios de televisión por cable y de valor agregado, la introducción del internet y de los servicios de Direct to Home $\mathrm{TV}$, son sólo algunas de las cuestiones que han venido a cambiar en cuestión de un par de años. ${ }^{14}$

Pese a que, en su momento voces de mexicanos que se alzaron para cuestionar la omisión clara que el TLC hace en referencia a los medios de comunicación y del crecimiento del sector de las telecomunicaciones, el marco jurídico correspondiente permaneció inamovible, a excepción de la reforma al Artículo 28 Constitucional que modifica la prioridad del Estado para intervenir en algunos sectores básicos entre ellos el de las telecomunicaciones, y la promulgación de la nueva Ley de Telecomunicaciones que se hiciera en 1995, la cual privatiza la industria y abre la posibilidad de que se de la participación de extranjeros en ese sector. ${ }^{15}$ Otra modificación indirecta al marco jurídico de las comunicaciones, pero que facultó la reestructuración del sector fue la nueva Ley de Inversiones Extranjeras enviada al Congreso en noviembre de 1993 y publicada en diciembre del mismo año. ${ }^{16}$ Estas y otras disposiciones relativas completaron el panorama de la posibilidad de aceptar inversión extranjera en renglones estratégicos de la economía nacional, entre ellos telefonía básica y televisión por cable. Entre las actividades reservadas a mexicanos o sociedades nacionales con exclusión a extran-

14 La televisión restringida por ejemplo, es un negocio cuya oferta supera ya a la demanda, pero para el cual se espera un nivel de rentabilidad superior a los mil millones de dólares en los próximos cuatro años. $E l$ Financiero, 4 de febrero de 1997., p.12

15 La Ley de Telecomunicaciones en su artículo 12 faculta la inversión extranjera en hasta un $49 \%$ de participación. "Ley Federal de Telecomunicaciones". Diario Oficial de la Federación 7 de junio de 1995.

16 "Ley de Inversión Extranjera". Diario Oficial de la Federación. 27 de diciembre de 1993. Recientemente el Secretario de Comercio Herminio Blanco mencionó que se estudia la modificación de dicha ley con el propósito de hacer más ágil el ingreso de capitales al país, para desrregular y simplificar sus operaciones. Ver: "Anuncia Herminio Blanco cambios a la Ley de Inversiones Extranjeras." en: La Jornada 17 de octubre de 1996., p. 42. 
El marcojuridico y la política de comunicación ...

jeros según el cuerpo del TLC se encuentra la transmisión privada de programas de radio, asi como la transmisión y repetición de programas de televisión distintos de las sociedades por cable, pero ello no excluye la inversión mientras que la concesión esté en manos mexicanas. ${ }^{17}$

Hacia la fecha de la firma del Tratado de Libre Comercio de América del Norte fueron adaptadas parcialmente otras leyes relativas a los medios audiovisuales, por ejemplo la Ley de la Industria Cinematográfica que originalmente databa de 1949 fue derogada en función de una nueva promulgada en diciembre de $1992,{ }^{18}$ mientras que la ley Federal de Radio y Televisión originalmente de 1960 no fue modificada. ${ }^{19}$

Las modificaciones a las legislaciones relativas a la comunicación y a otras actividades complementarias, aunque fundamentales pasaron desapercibidas en su momento, excepto para algunos especialistas, mientras las negociaciones en relación al Tratado de Libre Comercio de Norteamérica continuaban su marcha. Así, durante muchos meses, aún después de la entrada en vigencia del Tratado de Libre Comercio para América del Norte (TLC) se hacía todavía hincapié en que éste no afectaba a la operación fundamental de los medios de comunicación en México.

Una revisión exhaustiva, sin embargo, del cuerpo del texto del Tratado de Libre Comercio para América del Norte, asi como de sus anexos, especialmente en las listas de especificaciones relativas a México, arrojan datos contundentes respecto del tipo de medios o servicios de comunicación implicados que se reformulan en su operación a la luz de lo que el TLC faculta. (Ver Anexo Lista de México). Resulta evidente entonces que la relación trilateral con Estados Unidos y Canadá implica para México en materia

17 Además, la nueva Ley de Inversiones Extranjeras prevé por ejemplo, que en el primer año de vigencia los capitales extranjeros pueden adquirir empresas nacionales, sin autorización de la Comisión Nacional de Inversiones Extranjeras hasta por un valor menor a los 25 millones de dólares en sectores no reservados, hasta llegar a los 150 millones de dólares en el décimo año. El capital internacional podrá adquirir una compañía nacional por un monto superior al establecido cada año, pero mediante aprobación de la Comisión Nacional de Inversiones Extranjeras.

18 Se dice que ésta última fue promulgada "al vapor" con el fin de adecuar la industria cinematográfica a las exigencias del Tratado de Libre Comercio de América del Norte que estaba en proceso de negociación. Esto no sucedió con la Ley Federal de Radio y Televisión debido a que el crecimiento del sector y las posibilidades de crecimiento se veian venir mayormente en el área de las telecomunicaciones y de la televisión restringida que en la televisión abierta, lo cual estaba siendo directamente contemplado por la Ley de Telecomunicaciones que cristalizó en junio de 1995.

19 La última modificación a esta ley se registra en en enero de 1986. 
de comunicaciones mucho más de lo que los negociadores en su momento quisieron dejar entrever.

Ahora bien, el cuerpo del texto del TLC es un buen instrumento para conocer el resultado final de las negociaciones: no asi la base sobre la cual negoció cada país.

En el caso de México, es importante hacer notar que nuestros marcos legales en materia de medios estuvieron siempre detrás del contexto negociado. Por ello es muy importante indicar cuál es el estado que guarda nuestro marco legislativo en esa materia.Siendo la legislación el marco jurídico bajo el cual operan los medios, sería lógico pensar que debieran adecuarse al ritmo de los tiempos, pero en realidad esto no sucede. Dicho de otra manera, por distintos motivos, por no coincidir con el calendario de los tiempos legislativos destinados a la discusión, o por otras razones, ${ }^{20}$ la estructura jurídica que rige a los sistemas de comunicaciones mexicanos no se ha modificado y por lo tanto ha dejado una puerta abierta a la propia industria quien ha comenzado a crecer caótica y desmesuradamente.

En el recinto legislativo se ha debatido sobre el particular; lamentablemente no se ha logrado la aprobación de un proyecto de ley específico que integre todos los ámbitos de la comunicación social, incluyendo a las nuevas tecnologías. ${ }^{21}$

Un signo alentador, sin embargo es el hecho de que la problemática de los medios de comunicación sea discutida abietamente dentro del recinto legislativo. Nunca antes en la historia reciente de México se habían puesto sobre la mesa tantos cuestionamientos en relación a los regímenes juridicos imperantes en el sistema de medios, sencillamente porque la legislación era si no suficiente por lo menos medianamente funcional para

20 Según informaciones dadas a conocer por la prensa, la Comisión Especial de Comuniación Social integrada por los distintos partidos con representatividad en la Cámara de Diputados tiene listo ya un anteproyecto de Ley de Comunicación Social, sólo falta discutirlo con el Ejecutivo para que éste lo envíe formalmente a la consideración del Poder Legislativo. Desafortunadamente, por una cosa o por otra el Presidente Zedillo no ha podido recibir a los legisladores, "quienes a lo largo de 16 semanas le han pedido una entrevista sin que hasta el momento hayan sido atendidos. La Jornada 18 de septiembre de $199, p)$.

21 El anteproyecto de ley debe enviarse a tiempo para su discusión dentro de la L VI Legislatura, de otra forma pudiera correrse el riesgo de posponer su promulgación. De acuerdo con [la Diputada María Teresa Gómez Mont, miembro de la Comisión y\} legisladora panista, el retraso del Ejecutivo en el proceso no es más que un reflejo de que dentro del gobierno hay sectores que no quieren llegar a una reforma en materia de comunicación social, y que amparados en un discurso que habla de libertad de expresión, pretenden en realidad que no se legisle, con el fin de mantener esos sistemas de control informativo que se han generado durante mucho tiempo, de mantener los privilegios, de comprar conciencias, y sobre todo de no cambiar". La Jornada, 18 de septiembre de 1996., p. 7. 
regular al sector. Sin embargo, los grandes cambios tecnológicos junto con la entrada del TLC a nuestro país han obligado a los legisladores a replantease el problema.

Una de las formas en que en los últimos años el sistema político ha integrado las demandas de los distintos sectores sociales en relación al crecimiento desmesurado de los sistemas de comunicación ha sido a través de los foros de consulta. México no tiene, como Canadá una larga tradición en este tipo de esquemas de participación política, no obstante algunas propuestas se han hecho llegar en distintas oportunidades hasta la tribuna legislativa más alta del país. Nuestro país ha tenido diversas experiencias en este sentido, la primera de ellas en la década de los ochentas, en la cual el gobierno del Presidente José López Portillo organizó los primeros Foros de Consulta Popular de Comunicación Social. ${ }^{22}$

También se han llevado a cabo foros de consulta como parte de la creación de los distintos planes sexenales de gobierno; por ejemplo en el último de ellos convocado por el gobiemo del Presidente Emesto Zedillo, ${ }^{23}$ se hicieron múltiples análisis, evaluaciones y críticas que dejaron como resultado el clamor de algunos sectores que pidieron entonces la modificación urgente de las leyes y reglamentos de la radio y la televisión, de imprenta y de cinematografia, puesto que, según se dijo, ya no responden a las necesidades básicas que enfrenta el país. Asimsmo, se pidió la creación de un Consejo Plural de Comunicación Social con estatuto de organismo público y personalidad jurídica, integrado por profesionales de la comunicación, organismos públicos, sociales, privados y culturales para supervisar la aplicación rigurosa de las diversas leyes en materia de comunicación colectiva. También se solicitó modificar los criterios de asignación de concesiones para el uso de frecuencias de radio y televisión, asi como garantizar el uso público de los tiempos del Estado. ${ }^{24}$

Entre los foros de consulta pública sobre la materia llevados a cabo más recientemente se encuentran los convocados por dos comisiones legislativas actualmente en funciones. Una de ellas, la Comisión Especial de Comunicación Social y la otra, la Comisión de Cultura, ambas pertenecientes a la LVI Legislatura de la Cámara de

22 La puesta en marcha de dicho proceso de consulta data del 24 de marzo de 1983, siendo coordinador general de los foros el Lic. Manuel Bartlett Díaz.

23 Plan Nacional de Desarrollo 1995-2000, se llevó a cabo un foro denominado Foro de Consulta Popular sobre Estado, democrar ; y medios de comunicación (28 de abril de 1995), en el que participaron entre otros sectores: medios ، .omunicación, académicos y partidos políticos.

24 Por ley los concesionarios de la radio y la televisión están obligados a proporcionar el $12.5 \%$ del tiempo de transmisión para usos del Estado. 
Diputados. Aunque la Comisión de Cultura se refirió más bien a la problemática de las instituciones encargadas de la difusión del arte y la cultura en México también tomó en consideración la problemática de los medios de comunicación; fue sin embargo la Comisión Especial de Comunicación Social la que mayormente abordó el problema.

La Comisión de Cultura, se abocó a los siguientes temas: Producción cultural en medios audiovisuales, derechos de autor, cultura indígena y cultura popular, educación artística e investigación en y para la cultura, promoción de la lectura, patrimonio material e intangible de la nación, infraestructura, centros comunitarios y regionalización de la cultura, el papel de la sociedad civil en la cultura, promoción y difusión de la cultura y empresas culturales y estímulos a la creación artística.

La Comisión Especial de Comunicación Social en cambio, trató directamente la problemática de los medios de comunicación en México. No obstante, pese a lo avanzado de la temática de la consulta y a la diversidad de las aportaciones, el trabajo de ambas comisiones hasta este momento no ha dado por resultado la transformación de las estructuras jurídicas y de los marcos legales correspondientes. ${ }^{25}$

En este trabajo se toman en consideración las temáticas generales que tocan ambas comisiones pero particularmente la Comisión Especial de Comunicación Social. Posteriormente se hace una referencia a los resultados a los que han llegado los últimos foros de consulta pública tanto en México como en Canadá a la luz de la problemática del Tratado de Libre Comercio de América del Norte.

La Comisión Especial de Comunicación Social de la Cámara de Diputados, fue creada el 28 de enero de 1995 y quedó formalmente instalada el 8 de febrero del mismo año con el mandato de consultar a la ciudadanía y recoger opiniones de los especialistas con el propósito de adecuar el marco jurídico a las transformaciones ocurridas en el ámbito de la información y de la comunicación. Dicha Comisión estuvo integrada por 35 diputados de los cuatro grupos parlamentarios representados en la Cámara. De ellos 20 legisladores pertenecen al Partido Revolucionario Institucional (PRI), 9 al Partido

25 La Comisión de Comunicación Social de la Cámara de Diputados informó que después de varias semanas de intentar reunirse con el Secretario de Gobernación Emilio Chuayfet, finalmente se presentaría un paquete de iniciativas para reformar los artículos sexto y séptimo de la Constitución con el fin de garantizar el derecho a la información; modificar las leyes de radio, televisión y cinematografia y derechos de autor, asi como para crear tres nuevas leyes, de libros, de publicidad y de informática. La Jornada. 18 de octubre de 1996., p.3. Al momento de llevar a cabo este trabajo todavía no se conocían los resultados de dicha reunión. 
El marco jurídico y la política de comunicación ...

Acción Nacional (PAN), 4 al Partido de la Revolución Democrática (PRD) y 1 al Partido del Trabajo (PT), asi como por un diputado independiente.

Esta Comisión abordó el problema de la comunicación social convocando a foros de consulta sobre el problema. La consulta tuvo lugar en diez ciudades del país del 8 de junio al 11 de julio de 1995.

Los temas abordados por la consulta fueron: el derecho a la información, prensa escrita, radio, cine, televisión, edición y distribución de discos y videos, teatro, espectáculos, satélites, régimen de concesiones, publicidad, informática, sistema de telecomunicaciones, comunicación entorno ecológico y urbano, nuevas tecnologías y relación Estado-medios de comunicación.

En la consulta pública en materia de comunicación social llevada a cabo en el verano de 1995 fueron presentadas 2,908 propuestas en 10 foros en las siguientes ciudades: Distrito Federal, Mérida, León, Puebla, Oaxaca, Guadalajara, Zacatecas, Hermosillo, Monterrey y Tijuana. Posteriormente se recibieron 37 trabajos en otros foros académicos y especializados.

Los trabajos se presentaron en mesas con la siguiente temática:

- Mesa uno: Estado, sociedad y medios de comunicación

- Mesa dos: Medios electrónicos

- Mesa tres: Medios impresos

- Mesa cuatro: Programación, agencias de noticias y publicidad

- Mesa cinco: Propiedad intelectual y Derechos de autor

- Mesa seis: Espectáculos, espacios culturales y recreativos.

Debido a que el Tratado de Libre Comercio para América del Norte posibilita la participación de particulares nacionales y extranjeros en varios rubros, incluído el renglón de las telecomunicaciones, ${ }^{26}$ un tema muy importante que fue retomado por los participantes en los foros de consulta fue el de la soberanía nacional.

26 Tratado de Libre Comercio de América del Norte. Tomo II. Anexo I. Lista de México. Para el caso de México el texto del TLC en el anexo antes mencionado, estable que los servicios que pueden recibir participación extranjera hasta en un $49 \%$ son además de los servicios telefónicos básicos, telefonía rural y telefonía celular, servicios satelitales, servicios de localización, telefonia móvil, télex, videotexto y servicios de información, radio, televisión aérea, televisión por cable, televisión de alta definición, sistemas de distribución multipunto y cine. 
Asi pues los aspectos más importantes relacionados con medios audiovisuales y específicamente relacionadas con la problemática del Tratado de Libre Comercio de América del Norte fueron los siguientes:

1. El Estado mexicano debe asumir su papel de rector y orientador del sistema de comunicación social sobre todo en función de la entrada en vigor del Tratado de Libre Comercio para Norteamérica.

2. Debe recuperarse la soberanía nacional sobre el espacio aéreo mexicano, lo cual implica revisar el TLC en su apartado de la materia a fin de reservar a los particulares nacionales el uso de las ondas electromagnéticas que viajan sobre el espacio aéreo nacional.

3. Deben promoverse nuevas disposiciones dentro del TLC a fin de garantizar el respeto a los Derechos de Autor.

Frente a todo lo anterior, una conclusión muy importante que emanó de los participantes a los foros de consulta fue la necesidad de integrar una política sólida y coherente de información y medios de comunicación como mecanismo de defensa ante los ímpetus de la globalización y la transnacionalización.

Se reconoció asimismo que la globalización no es únicamente económica, sino que su impacto cultural es considerable principalmente a través de los medios electrónicos como la radio y la televisión, por lo tanto se solicitó a la legislatura buscara crear nuevos canales de comunicación como mecanismo para la defensa de la identidad nacional mexicana. En tomo a ello, se elaboraron las siguientes propuestas:

1. Abrir nuevos canales para que las diversas agrupaciones y organizaciones sociales puedan expresarse mediante la radio y la televisión, salvaguardando así nuestra idiosincracia, idioma y cultura.

2. Fomentar la radio y la televisión comunitarias, creando espacios para la difusión de la gran diversidad de grupos étnicos y sociales que conforman la nación mexicana, satisfaciendo así sus necesidades de expresión e información.

3. Promover los patrones de la cultura nacional en los medios de comunicación, fortaleciendo y acrecentando la identidad y la soberanía nacionales.

En lo referente a los marcos jurídicos actuales que regulan la actividad tanto del cine como de los medios electrónicos, fue casi unánime la petición de los participantes a los foros de consulta en el sentido de que deben actualizarse. 
En lo que corresponde a las actividades de la radio y a la televisión se hizo un especial énfasis en los siguientes rubros:

a) Replantear el otorgamiento de concesiones en materia de radio y televisión. ${ }^{27}$

b) Remover las facultades unilaterales del Ejecutivo Federal en el otorgamiento de concesiones turnándolas al Poder Legislativo.

c) Promover la creación de una Comisión o Consejo encargado de vigilar, evaluar y coordinar la actividad de los medios electónicos.

d) Incorporar a la legislación la regulación de nuevas tecnologías tales como las fibras ópticas, televisión interactiva, televisión de alta definición, internet, CD-ROM y otras.

e) Fortalecer la identidad nacional mexicana por medio de programas que difundan la cultura y los valores nacionales, promoviendo el fortalecimiento de los valores cívicos, familiares y el respeto a los semejantes.

En lo que corresponde a la cinematografía los participantes a los foros de consulta propusieron modificar la Ley de la Industria Cinematográfica en los siguientes aspectos:

a) Crear una nueva ley de cinematografía donde participen los cineastas y lo que es más, formular una ley federal de comuniación social que abarque a todos los medios de comunicación.

b) Promover la producción y la distribución de material fílmico nacional ${ }^{28}$ y buscar nuevas formas de financiamiento para la producción de material fílmico en el país.

c) Crear una cultura cinematográfica que exalte los valores de la idiosincracia y rescate los valores históricos y humanos de los mexicanos.

27 La ley federal actual establece que las concesiones para radio y televisión se otorgarán por un periodo de 30 años y podrá ser refrendada al mismo concesionario que tendrá preferencia sobre terceros. (Ley Federal de Radio y Televisión. Artículo 16, enero de 1960). Los ciudadanos participante en los foros de consulta coinciden en establecer que la duración del término de las concesiones es excesiva; se sugieren por ejemplo 15 años y que para evitar monopolios, se fije un máximo de tres concesiones o permisos asignados a una misma persona o sociedad. Además, que la sociedad pueda tener conocimientos claros y completos del proyecto de inversión, de los propósitos generales del solicitante.

28 La ley de cinematografia vigente fue recientemente promulgada para adecuarse a los requerimientos del TLC, los cineastas establecen que dicha ley no protege a la cinematografia nacional, ya que además de otorgar la posibilidad de una mayor participación de inversión extranjera, no favorece la exhibición de material nacional y tampoco protege suficientemente los derechos de autor de cineastas mexicanos. (Ley Federal de Cinematografía. Arts. 11 y 16. diciembre de 1992). 
Un reconocimiento importante que los participantes hicieron durante los foros de consulta sobre comunicación fue el que las nuevas tecnologias informativas están produciendo una integración de los campos de la comunicación y la informática y que las nuevas tecnologías inciden directamente en los medios de comunicación actuales. Por otro lado, también se reconoció que se trata éste de un campo no regulado, que está creciendo con rapidez pero de forma anárquica, lo cual da lugar a diversas transgresiones y delitos informáticos.

Dentro del ámbito del TLC además, se planteó la necesidad de revisar la Ley Federal de Telecomunicaciones y las otras relativas (Ley Federal de Radio y Televisión, Ley de la Industria Cinematográfica y Ley Federal de Derechos de Autor) en virtud de que las nuevas tecnologias dan lugar a nuevas actividades relacionadas con la informática y las telecomunicaciones que no se encuentran contempladas en las legislaciones originales.

Por otra parte, es importante mencionar que además se contemplaron cuestiones como el acceso a las nuevas tecnologías, las nuevas tecnologías y la educación, las nuevas tecnologías y el desarrollo nacional y la necesidad de hacer investigación sobre nuevas tecnologías, cuestiones todas que no constituyen parte integral de los cuerpos jurídicos existentes.

Los participantes formularon la petición expresa a los legisladores de incorporar a México a los vertiginosos cambios que viven las telecomunicaciones mundiales, pero garantizando la homologación y la integración de alternativas en materia de telecomunicaciones o teleinformática de manera que México pueda competir eficientemente en el "concierto de la globalización informativa".

Es importante hacer notar, que si bien en el presente trabajo se intenta una recopilación de las diversas posturas puestas de manifiesto en los distintos foros, el sector de los medios de comunicación como en muchos otros de la sociedad mexicana hay múltiples intereses que impiden ver claramente cuál puede ser la mejor alternativa para México; es por ello que, dependiendo de los actores involucrados y de las presiones que ejerzan la industria irá paulatinamente moviendose en ciertas direcciones finalmente al amparo de una legislación poco clara.

No obstante el panorama pudiese ser alentador en este sentido: tanto la historia reciente como las condiciones estructurales propias del sistema político mexicano hacen que convocar y atender a foros de consulta pública sean una experiencia novedosa. La consulta ha probado ser de mucha utilidad, sobre todo porque los grupos directamnte interesados en la actividad de la comunicación social han proporcionado propuestas muy concretas para actualizar el marco jurídico existente y en ese sentido representan un gran 
El marco jurídico y la política de comunicación ...

paso hacia la democratización. Sin embargo los tiempos políticos parecen no ser los idóneos para transformar las estructuras; en este momento a juicio del gobierno lo que se necesita es que el país se desarrolle y que ingresen capitales al país, y si ello significa abrir mucho más el sector, lo mas probable es que se siga haciendo. Todo dependerá en los próximos meses de la tenacidad de los grupos de presión ya que un sistema de comunicaciones moderno también requiere de un marco jurídico justo y equitativo para todos los involucrados.

Canadienses y mexicanos se enfrentan a una misma problemática: la presencia de contenidos extranjeros, pero con diferentes herramientas operativas e infraestructura; la de Canadá se encuentra plenamente consolidada y ha entrado en una fase selectiva de crecimiento, ${ }^{29}$ la de México, todavía en el desorden, se está conformando a partir de una serie de posibilidades de crecimiento tecnológico que se encuentran apenas en fase de implementación. Los recursos pues, con los que ambos paises se enfrentan a un fenómeno complejo y cambiante, son distintos: Canadá ha venido reforzando la producción de programación nacional a partir de financiamientos federales y privados mientras que México padece una crisis de producción nacional debido a la reorientación general del sector de las comunicaciones hacia la privatización y la importación de contenidos. ${ }^{30}$

La palabra clave pareciera ser acceso. Este término parece ser el único común denominador entre aquellos que desean liberar la lindustria de las comunicaciones para hacerla más competitiva y aquellos que desean proporcionar una oferta equilibrada de contenidos nacionales y extranjeros. Canadá comulga con la idea que el excedente en la oferta de comunicación por parte de industrias extranjeras debe de ser contrarrestado con programación nacional, de manera que el consumidor canadiense tenga acceso a una variada mezcla de programación propia que le permita afianzar los valores de su

Q

29 A resultas de los cambios en el ámbito internacional y de la estructura de bloque a la que se adhiere Canadá primero bilateralmente con Estados Unidos y luego a través del TLC, el sector de las comunicaciones se liberalizó y en ello jugó una parte importante la CRTC, sin embargo la comisión canadiense ahora se enfrenta a la problemática de conciliar intereses de actores industriales e incluso de receptores que todavia se encuentran en la mesa de las discusiones. A este respecto ver Wilson, Kevin. "Liberalización de las telecomunicaciones en Canadá". en: Delia Crovi (Coordinadora). Desarrollo de las industrias audiovisuales en México y Canadá. Facultad de Ciencias Políticas y Sociales, UNAM, 1995., pp. 233-255.

30 Un desarrollo pormenorizado de los cambios que ha sufrido el sector se encuentran detallados en: Casas Pérez, María de la Luz. "Estructura para el análisis de la reglamentación mexicana en medios audiovisuales", en: Delia Crovi (coordinadora). Desarrollo de las industrias audiovisuales en México y Canadá. Proyecto Monarca. Facultad de Ciencias Políticas y Sociales, UNAM, 1995., pp. 65-79. 
identidad nacional. ${ }^{31}$ No obstante, Canadá mismo está experimentando una lucha interna entre los intereses de los empresarios o industriales de la comunicación y aquellos que todavía desean diseñar una política que defienda los usos sociales de los medios. ${ }^{32}$ Todavía no es posible saber quién saldrá victorioso en esa lucha.

México, por su parte experimenta su propia lucha intema: hasta hace unos años, el tema de nuestra integración cultural al bloque económico de América del Norte a través de los medios de comunicación, era debatido exclusivamente en círculos académicos e intelectuales, poco o nada llegaba de estas reflexiones a la máxima tribuna del país. Hoy en día por primera vez en la historia jurídica reciente de este país se está llevando a cabo un esfuerzo por considerar integralmente a la problemática de los medios mexicanos y cristalizar su operación en una política integral que tome en consideración a la identidad nacional y a la cultura. No obstante también está por verse quién saldrá victtorioso de ese debate, si aquellos sectores de la sociedad que luchan por hacer de los medios auténticos vínculos de comunicación entre todos los mexicanos, o quienes desean acelerar el crecimiento del sector sencillamente porque ello significa una mayor oferta de las comunicaciones.

31. En términos generales los estudios recientes sobre el contenido de los medios y la identidad cultural se dividen en dos vertientes fundamentales: una que vincula la presencia de contenidos extranjeros en la comunicación a un debilitamiento de la identidad cultural (Beltrán et al,1980, 1981), más recientemente Esteinou (1990) y Casas (1994), y otra que establece que debido a la apropiación e interpretación que hacen los receptores de los contenidos, la exposición a la oferta programática no es equivalente a la pérdida de la identidad cultural(Sepstrup,1995), (Lozano, 1995); sin embargo, también hay que tomar en consideración que si bien en este momento la programación importada no es la que más teleauditorio tiene, esto puede cambiar con consecuencias culturales más o menos amplias (Sánchez Ruiz, 1995).

32 En última instancia será el receptor como usuario de los servicios de comunicaciones, quien tendrá en sus manos la discusión de la política pública de comunicación en los próximos años. Por el momento los suscriptores domésticos desean recibir más por lo que pagan, (recordemos que en el caso de Canadá todos los servicios, incluyendo la cabledistribución son de paga, mientras que en el caso de México todavía tenemos el fenómeno de la televisión abierta), pero existen grupos que se han empeñado en recordarle a la CRTC las especificaciones de su mandato. Al respecto ver: Wilson, Kevin. "Liberalización de las telecomunicaciones en Canadá". en: Delia Crovi (Coordinadora). Desarrollo de las industrias audiovisuales en México y Canadá. Proyecto Monarca. Facultad de Ciencias Políticas y Sociales, UNAM, 1995., pp. 233-255. 


\section{Conclusiones}

El aspecto del diseño de políticas públicas y particularmente el de la reglamentación en medios es frecuentemente considerado como uno de los más áridos y regularmente desatendidos de la investigación. El consenso general, por lo menos en el caso de México, es que el proceso de actualización legislativa generalmente marcha a la zaga de los cambios a los que nos arrastra la tecnología.

La apertura de México hacia los mercados internacionales, la reestructuración sufrida al interior del sector de las comunicaciones y nuestra vinculación con el bloque económico de América del Norte a raíz de la firma del TLC nos obligan nuevamente a reconsiderar este aspecto por ser uno de los pilares sobre los cuales se desarrollará la actividad de las comunicaciones para los próximos años y el próximo siglo.

La posibilidad de observar el caso de nuestros amigos y socios comerciales en Canadá nos permite reflexionar acerca de una problemática que nos es común y que nos compromete, esto es, la vinculación que tenemos a través del TLC con nuestro tercer socio comercial que es los Estados Unidos de Norteamérica y el flujo cada vez más expedito de información entre los tres países.

La forma en que tanto México como Canadá han reaccionado en el ámbito de las políticas públicas, particularmente las de comunicación en materia de medios audiovisuales y telecomunicaciones son centrales para entender la relación, incluso política, que cada uno de los dos países entablará con su vecino común. Hay que recordar, sin embargo, que la política escrita frecuentemente contrasta con la política práctica en el sentido de que los documentos oficiales hablan de la postura gubernamental supuestamente articulando los intereses de todos los actores involucrados, pero frecuentemente ocultan las disputas y las negociaciones que se dan tras bambalinas.

En ese sentido es que estre trabajo se ha dado a la tarea de abordar, por un lado los documentos oficiales que norman la actividad del sector, y por otro las preocupaciones expresadas a nivel de consulta pública sobre el particular, que si bien no son exhaustivos en el sentido de explorar todo el panorama de opiniones y criterios que se han puesto de manifiesto ante lo vertiginoso de los cambios, sí son material de estudio y consulta por parte de los cuerpos legislativos que habrán de plasmar en una política definitiva la operación de los medios en el futuro. 


\section{Bibliohemerografía}

Cámara de Diputados. Comisión Especial de Comunicación Social. Consulta Pública en Materia de Comunicación Social. Primera Etapa: Relatoria Foros Regionales de Consulta. (Versión Preliminar). 25 de octubre de 1995.

Canada. Canadian Radio-television and Telecommunications Commission. Competition and Culture on Canada's Information Highway. 19 May, 1995.

CASAS-PÉrEZ, María de la Luz. Análisis del ámbito socio-politico en el que se enmarca una posible politica cultural de comunicación, ante la perspectiva del acuerdo tri-lateral México-Estados Unidos-Canadá. Tesis doctoral. UNAM, 1994.

CONNOR, Edward. "The Department of Communications Under Free Trade Regime". Canadian Journal of Communication. Volume 16., Number 2, 1991.

CROvi, Delia. (Coordinadora). Desarrollo de las industrias audiovisuales en México y Canadá. Facultad de Ciencias Políticas y Sociales, UNAM, 1995.

EsTEINOU MADRID, Javier. "El peso del Tratado de Libre Comercio sobre la cultura nacional". Revista Mexicana de Comunicación. Año cinco, Número veintisiete, enero-febrero de 1993.

FulLER, Colleen. "Fade to Black: Culture Under Free Trade". Canadian Forum, August, 1991.

JALIFE DAHER, Mauricio y José Luis Caballero Leal. "Proyecto de una nueva Ley de Derechos de Autor: un rumor engargolado".El Financiero. 15 de octubre de 1996.

Secretaría de Comercio y Fomento Industrial. Tratado de Libre Comercio de América del Norte. México, 1992.

Secretaría de Comercio y Fomento Industrial. "Ley de Inversión Extranjera". Diario Oficial de la Federación. 27 de diciembre de 1993.

Secretaría de Comunicaciones y Transportes. "Ley Federal de Telecomunicaciones". Diario Oficial de la Federación 7 de junio de 1995.

Secretaría de Educación Pública. "Ley Federal de Derechos de Autor". Diario Oficial de la Federación. 22 de diciembre de 1993.

Secretaria de Gobernación. "Lcy Fcdcral de Cinematografía". Diario Oficial de la Federación. 23 de diciembre de 1992

Secretaría de Gobernación. "Ley Federal de Radio y Televisión". Diario Oficial de la Federación. 19 de enero de 1960. 
El marco juridico y la politica de comunicación ...

TREMBLAY, Gaëtan et Jean-Guy Lacroix. Le Projet Monarque. Étude comparée des industries québécoises et mexicaines de láudiovisuel. Collection Documents GRICIS. Université du Québec à Montréal, 1995. 


\section{Apéndice 1}

\section{Contenido del texto del Tratado de Libre Comercio para América del Norte} relativo a medios de comunicación

\begin{tabular}{|c|c|c|c|}
\hline Sector y subsector & $\begin{array}{l}\text { Acciones que faculta el } \\
\text { tratado }\end{array}$ & Medios implicados & $\begin{array}{l}\text { Sección de } \\
\text { referencia }\end{array}$ \\
\hline $\begin{array}{l}\text { Sector: } \\
\text { Comunicaciones } \\
\text { Subsector: } \\
\text { Telecomunicaciones }\end{array}$ & $\begin{array}{l}\text { Participación extranjera } \\
\text { hasta del } 49 \% \text { en } \\
\text { servicios de videotexto y } \\
\text { servicios mejorados de } \\
\text { conmutación de } \\
\text { paquetes. } \\
\text { México se reserva el } \\
\text { derecho de adoptar o } \\
\text { mantener cualquier } \\
\text { medida relativa a la } \\
\text { inversión de estos } \\
\text { servicios. } \\
\text { Las actividades de } \\
\text { radiotelegrafia y } \\
\text { comunicación via satélite } \\
\text { quedan reservadas al } \\
\text { Estado mexicano. } \\
\text { Participación extranjera } \\
\text { en transferencia de } \\
\text { tecnología y } \\
\text { financiamiento; México } \\
\text { podrá subarrendar el } \\
\text { excedente de su } \\
\text { capacidad de } \\
\text { infraestructura hasta por } \\
\text { un } 30 \% \text { de su capacidad } \\
\text { instalada para fines de } \\
\text { red privada. }\end{array}$ & \begin{tabular}{|l|} 
Servicios telefónicos \\
básicos; telefonía \\
rural y telefonía \\
celular; servicios \\
satelitales; servicios \\
de localización; \\
telefonia móvil; \\
télex; videotexto y \\
servicios de \\
información; (prensa \\
y publicaciones \\
periódicas quedan \\
implicadas por el \\
uso de estas \\
tecnologías.
\end{tabular} & $\begin{array}{l}\text { Tomo II. } \\
\text { Anexo I. } \\
\text { lista de México. } \\
\text { Tomo II. } \\
\text { Anexo II. } \\
\text { Lista de México. } \\
\\
\text { Tomo II. } \\
\text { Anexo III. } \\
\text { Lista de México. } \\
\\
\text { Tomo II. } \\
\text { Anexo V. } \\
\text { Lista de México. }\end{array}$ \\
\hline
\end{tabular}


El marco jurídico y la política de comunicación ...

\begin{tabular}{|c|c|c|c|}
\hline Sector y subsector & $\begin{array}{l}\text { Acciones que faculta el } \\
\text { tratado }\end{array}$ & Medios implicados & $\begin{array}{l}\text { Sección de } \\
\text { referencia }\end{array}$ \\
\hline $\begin{array}{l}\text { Sector: } \\
\text { Comunicaciones } \\
\text { Subsector: } \\
\text { Servicios de } \\
\text { Esparcimiento }\end{array}$ & $\begin{array}{l}\text { Se requiere autorización } \\
\text { de la Secretaría de } \\
\text { Gobernación para } \\
\text { retransmitir o distribuir } \\
\text { programas desarrollados } \\
\text { en el extranjero, previo } \\
\text { pago de derechos de } \\
\text { retransmisión. } \\
\text { La misma Secretaría } \\
\text { podrá autorizar el uso de } \\
\text { otro idioma que no sea } \\
\text { español. La publicidad } \\
\text { extranjera no puede ser } \\
\text { retransmitida. } \\
\text { Los insversionistas } \\
\text { extranjeros pueden } \\
\text { participar hasta en un } \\
49 \% \text { de empresas de } \\
\text { esparcimiento. } \\
\text { Se requiere la } \\
\text { autorización de la } \\
\text { Secretaría de } \\
\text { Comunicaciones y } \\
\text { Transportes para la } \\
\text { instalación y operación } \\
\text { de nuevos servicios de } \\
\text { televisión por cable. La } \\
\text { conexión será otorgada a } \\
\text { mexicanos. El } 30 \% \text { de } \\
\text { tiempo anual en pantalla } \\
\text { de cada sala } \\
\text { cinematográfica está } \\
\text { reservado a }\end{array}$ & $\begin{array}{l}\text { Radio; Televisión } \\
\text { aerea; televisión por } \\
\text { cable; televisión de } \\
\text { alta definición; } \\
\text { sistemas de } \\
\text { distribución } \\
\text { multipunto y cine. }\end{array}$ & $\begin{array}{l}\text { Tomo II. } \\
\text { Anexo I. } \\
\text { Lista de México. }\end{array}$ \\
\hline
\end{tabular}




\begin{tabular}{|c|c|c|c|}
\hline Sector y subsector & $\begin{array}{l}\text { Acciones que faculta } \\
\text { el tratado }\end{array}$ & Medios implicados & Sección de referencia \\
\hline $\begin{array}{l}\text { Sector: } \\
\text { Comunicaciones } \\
\text { Subsector: } \\
\text { Servicios de } \\
\text { Esparcimiento }\end{array}$ & $\begin{array}{l}\text { México se reserva el } \\
\text { derecho de adoptar } \\
\text { cualquier medida con } \\
\text { respecto a inversión } \\
\text { en servicios de } \\
\text { radiodifusión. } \\
\text { Sistemas de } \\
\text { distribución } \\
\text { multipunto y } \\
\text { televisión de alta } \\
\text { definición excepto en } \\
\text { los casos de } \\
\text { producción, venta o } \\
\text { derechos de } \\
\text { programas de radio o } \\
\text { televisión. } \\
\text { Los distribuidores de } \\
\text { películas producidas } \\
\text { fuera de México } \\
\text { donarán a la Cineteca } \\
\text { Nacional por cada } \\
\text { cinco títulos de } \\
\text { películas importadas, } \\
\text { una copia de dos } \\
\text { títulos de tales } \\
\text { películas. }\end{array}$ & $\begin{array}{l}\text { Radio; televisión } \\
\text { aerea; televisión por } \\
\text { cable; televisión de } \\
\text { alta definición; } \\
\text { sistemas de } \\
\text { distribución } \\
\text { multipunto; cine y } \\
\text { producción de videos, } \\
\text { audiocassettes y } \\
\text { música grabada. }\end{array}$ & $\begin{array}{l}\mid \\
\\
\text { Tomo II. } \\
\text { Anexo VI. } \\
\text { Lista de México. }\end{array}$ \\
\hline $\begin{array}{l}\text { Sector: } \\
\text { Servicios educativos. } \\
\text { Subsector: } \\
\text { Escuelas privadas. }\end{array}$ & $\begin{array}{l}\text { Faculta la inversión } \\
\text { extranjera por arriba } \\
\text { del } 49 \% \text { de } \\
\text { participación, previa } \\
\text { aprobación. } \\
\text { La autorización para } \\
\text { la prestación de } \\
\text { servicios se realizará } \\
\text { a la discreción de la } \\
\text { Secretaría de } \\
\text { Educación Pública o } \\
\text { de la autoridad estatal } \\
\text { correspondiente. }\end{array}$ & $\begin{array}{l}\text { En combinación con } \\
\text { medios y sistemas } \\
\text { tecnológicos } \\
\text { anteriores esto puedo } \\
\text { tener implicaciones } \\
\text { en sistemas } \\
\text { informativos y } \\
\text { didácticos, televisión } \\
\text { a distancia, } \\
\text { producción de obras } \\
\text { didácticas en video y } \\
\text { otros. }\end{array}$ & $\begin{array}{l}\text { Tomo II. } \\
\text { Anexo I. } \\
\text { Lista de México. } \\
\text { Tomo II. } \\
\text { Anexo V. } \\
\text { Lista de México. }\end{array}$ \\
\hline
\end{tabular}




\section{Apéndice 2}

Otros anexos del Tratado de Libre Comercio para América del Norte implicados con sectores de comunicación y/o cultura relativos a México.

La información que aparece a continuación, representa la lista de una Parte, de conformidad con los artículos 1108 (1), 1206819 y 1409(4), las reservas tomadas por una Parte con relación a medidas de disconformidad que derogan de una obligación sobre:

a) Trato nacional

b) Trato de la nación más favorecida

c) Presencia local

d) Requisitos de comportamiento

e) Requisitos de nacionalidad para los ejecutivos de alta dirección o miembros de juntas directivas

Anexo Lista de México

Sector: Comunicaciones.

Subsector: Servicios de Esparcimiento (Radiodifusión, Sistemas de distribución Multipunto o (MDS) u Televisión por Cable).

Clasificación Industrial: CMAP 941104 y CMAP 941105.

Descripción: Servicios Transfronterizos e Inversión.

Para proteger los derechos de autor, el concesionario de una estación comercial de radiodifusión o de un sistema de televisión por cable, requiere previa autorización de la Secretaría de Gobernación para retransmitir o distribuir en el territorio de México programas de radio o televisión desarrollados en el extranjero.

La autorización será concedida siempre que la solicitud lleve adjunta la documentación comprobatoria de el o los derechos para la retransmisión o distribución de tales programas.

Sector: Comunicaciones.

Subsector: Servicios de Esparcimiento (Limitada a Radiodifusión, Sistemas de Distribución Multipunto (MDS) y Televisión por Cable). 
Clasificación Industrial: CMAP 941104 y CMAP 941105.

Descripción: Servicios Transfronterizos e Inversión.

Se requiere del uso del idioma español para la transmisión, distribución por cable o en los sistemas de distribución multipunto, o programas de radio y televisión, excepto cuando la Secretaría de Gobernación autorice el uso de otro idioma.

La producción y programación que utilice la actuación de personal en vivo en actividades de radiodifusión deberá incluir un mayor tiempo cubierto por personas físicas de nacionalidad mexicana.

En México, los locutores y animadores de radio o televisión que no sean personas físicas de nacionalidad mexicana deberán obtener una autorización de la Secretaría de Gobernación para desempeñar dichas actividades.

Sector: Comunicaciones.

Subsector: Servicios de Esparcimiento (Transmisión y Sistemas de Distribución Multipunto (MDs) y Televisión por Cable).

Descripción: Servicios Transfronterizos e Inversión.

Se requiere el uso del idioma español o subtítulos en español en los anuncios radiodifundidos o distribuidos por cable en el territorio de México.

La publicidad incluida en los programas transmitidos directamente desde fuera del territorio de México no puede ser transmitida cuando los programas son retransmitidos o distribuidos en el territorio de México.

Sector: Comunicaciones.

Subsector: Servicios de Esparcimiento (Televisión por Cable).

Clasificación Industrial: CMAP 941105.

Descripción: Inversión.

Los inversionistas de otra Parte o sus inversiones sólo podrán adquirir, directa o indirectamente hasta un 49 por ciento de la participación en empresas establecidas o por establecerse en el territorio de México que posean o exploten sistemas de televisión por cable o que suministren servicios de televisión por cable.

Sector: Comunicaciones.

Subsector: Servicios de Esparcimiento (Televisión por Cable).

Clasificación Industrial: CMAP $\mathbf{9 4 1 1 0 5 .}$

Descripción: Servicios Transfronterizos. 
El marco jurídico y la política de comunicación ...

Se requiere una concesión otorgada por la Secretaría de Comunicaciones y Transportes para construir, administrary operar sistemas de televisión por cable. Tal concesión podrá ser otorgada sólo a personas físicas o morales de nacionalidad mexicana.

Sector: Comunicaciones.

Subsector: Servicios de Esparcimiento (Cines).

Clasificación Industrial: CMAP 941103.

Descripción: Servicios Transfronterizos e Inversión.

El treinta por ciento del tiempo anual en pantalla en cada sala, está reservado a las producciones mexicanas dentro o fuera del territorio de México.

Sector: Comunicaciones.

Subsector: Telecomunicaciones (Servicios Mejorados o de Valor Agregado).

Clasificación Industrial: CMAP 720006.

Descripción: Servicios Transfronterizos e Inversión.

1. Un proveedor de servicios mejorados o de valor agregado deberá obtener un permiso de la Secretaría de Comunicaciones y Transportes.

2. Las personas físicas o morales de Canadá o los Estados Unidos podrán proporcionar todos los servicios mejorados o de valor agregado, excepto el servicio de videotexto o el servicio mejorado de conmutación de paquetes, sin la necesidad de establecerse en el territorio de México.

3. Los servicios de videotexto y los servicios mejorados de conmutación de paquetes no podrán ser proporcionados de manera transfronteriza.

4. Los inversionistas de otra Parte o sus inversiones podrán adquirir el 100 por ciento de la participación en una empresa establecida o por establecerse en el territorio de México, para proporcionar cualquier servicio mejorado o de valor agregado, excepto los servicios de videotexto y los servicios mejorados de conmutación de paquetes.

5. Los inversionistas de otra Parte o sus inversiones podrán adquirir hasta el 49 por ciento de la participación en una empresa establecida o por establecerse en el territorio de México que proporcione servicios de videotexto y servicios mejorados de conmutación de paquetes. 
Sector: Comunicaciones.

Subsector: Transporte y Telecomunicaciones.

Clasificación Industrial: CMAP 7200; CMAP 7100 y CMAP 9411.

Descripción: Inversión.

Los gobiernos extranjeros y las empresas de Estado extranjero o sus inversiones no podrán invertir directa o indirectamente en empresas mexicanas que proporcionen servicios relacionados con las comunicaciones, el transporte, la radio y televisión y otras vías generales de comunicación, tal como se define en la Ley de Vías Generales de Comunicación. 